\title{
Evaluación del Aprendizaje Basado en Problemas en Estudiantes Universitarios de Construcciones Agrarias
}

\author{
César A. Rodríguez ${ }^{(1)}$ y José M. Fernández-Batanero(2) \\ (1 Departamento de Ingeniería Minera, Mecánica, Energética y de la Construcción, Universidad de Huelva, \\ Escuela Técnica Superior de Ingeniería, Campus La Rábida, Carretera Palos-Huelva s/n. 21819 Palos de \\ La Frontera, Huelva, España (e-mail: cesar@uhu.es) \\ (2) Departamento de Didáctica y Organización Educativa, Universidad de Sevilla, Facultad de Ciencias de \\ la Educación, C/ Pirotécnia s/n. 41013 Sevilla, España (e-mail: batanero@us.es).
}

Recibido May. 18, 2016; Aceptado Jul. 7, 2016; Versión final Ago. 26, 2016, Publicado Feb. 2017

\begin{abstract}
Resumen
En este trabajo se exponen los resultados de una aplicación y evaluación de la Metodología didáctica específica denominada Aprendizaje Basado en Problemas (ABP) en estudiantes de Construcciones Agrarias. El trabajo se ha efectuado durante el curso 2015-16 en dos grupos de alumnos del Grado de Ingeniería Agrícola de la Escuela Técnica Superior de Ingeniería de la Universidad de Huelva (España). El estudio incluyó la aplicación de la metodología didáctica específica y su evaluación. La evaluación se ha efectuado empleando un método cuasi-experimental. El diseño del ABP ha requerido una programación cuidadosa, incluyendo una parte significativa de trabajo autónomo por el estudiante. Los resultados muestran que el $\mathrm{ABP}$ es preferible a otra didáctica tradicional expositiva ya que se observa una diferencia significativa en la adquisición de conocimientos por los alumnos.
\end{abstract}

Palabras clave: aprendizaje basado en problemas; formación de ingenieros; método cuasi-experimental; construcciones agrarias

\section{The evaluation of a Problem-Based Learning approach in a graduate Agrarian Constructions program}

\begin{abstract}
This paper presents the results of a study into the implementation and evaluation of problem-based learning (PBL) methodology in an Agrarian Constructions program. The study was carried out during the academic year 2015-2016 with two groups of students of the degree in Agricultural Engineering of the School of Engineering at the University of Huelva in Spain. The study included the implementation of this novel approach and its evaluation. The evaluation was performed using a quasi-experimental method. Programming a course of PBL demands careful planning and the inclusion of a significant amount of selfdirected work by students. The results indicated that there is a significant difference in the acquisition of knowledge by students, suggesting that PBL should be preferred instead of the more traditional lecturebased approaches.
\end{abstract}

Keywords: problem-based learning; engineering education; quasi-experimental method; agrarian constructions 


\section{INTRODUCCIÓN}

El Aprendizaje Basado en Problemas (en adelante ABP) es una didáctica específica en la que el proceso de enseñanza y aprendizaje está caracterizado por el enfrentamiento de los alumnos a problemas más o menos complejos, reales las más de las veces, y para lo cual podrán disponer de cuanto material consideren necesario. Aunque en sus inicios el ABP comenzó a aplicarse a la enseñanza del Derecho, ha sido en las Facultades de Medicina donde ha encontrado tradicionalmente un desarrollo fructífero en un campo con fuerte implicación empírica y experimental (Barrows, 1985). Los precursores del ABP en medicina, Barrows y Tamblyn (1980) definen al ABP como "un método de aprendizaje basado en el principio de usar problemas como punto de partida para la adquisición e integración de los nuevos conocimientos" ( $p$. 1). La propuesta de la Escuela de Medicina de la Universidad de McMaster (Canadá), entre la década de los 60 y 70 , ha sido al respecto un antecedente clave en el desarrollo del ABP.

Desde entonces, el ABP ha evolucionado adaptándose a las necesidades de las diferentes áreas en las que fue aplicado, incluyendo las ingenierías y arquitectura. Todo ello ha implicado que se produzcan variaciones y adaptaciones con respecto a la propuesta original. Woods (2012) ha puesto precisamente el acento en mantener una estrategia enseñanza-aprendizaje coherente con los postulados de Barrows, rescatando el concepto de auténtico ABP que el desarrollador de la metodología en cuestión empleó para evitar confusión con otras metodologías que no eran realmente ABP. Sus elementos esenciales, no obstante, se mantienen. Un aprendizaje centrado en el alumno, el trabajo con grupos pequeños, el docente como facilitador, el autoconocimiento y los problemas como generadores de habilidades son comunes a la Metodología didáctica específica ABP (Rodríguez y Fernández-Batanero, 2016). En esta línea, los objetivos y tareas que se deben cumplir en el ABP son (Branda, 2009): utilizar estrategias de razonamiento para proponer hipótesis explicativas; identificar necesidades de aprendizaje, y capacitar para trasladar los aprendizajes conseguidos hacia otros problemas. Por su parte, y desde una perspectiva más flexible y adaptable, Felder y Brent (2009) insisten en que un aprendizaje activo (en sentido lato) es todo lo que no se ciña a ver, escuchar y tomar notas de clase. No obstante, insisten en que se debe seguir una estrategia de enseñanza que sea simple, eficaz, y que a su vez disponga de participantes con una base sólida en la investigación y el sentido común.

Actualmente, en el campo de la ingeniería se está implantando el ABP en numerosas materias. Tal como describe Fernández y Duarte (2013), existe una preocupación creciente por parte de las universidades y sus docentes por formar a ingenieros con las competencias que exige el entorno laboral. El acercamiento a la realidad que supone el ABP con respecto a otras didácticas tradicionales se hace patente. La aplicación del ABP a la ingeniería, no obstante, ha sido mucho más pausada con respecto al campo de la medicina. La forma de implantar un ABP presenta ciertos matices que deben ser tenidos en cuenta. Algunos trabajos aconsejan sobre la manera de implantar el ABP en el ámbito de la enseñanza de la ingeniería que nos ocupa en particular (Shinde e Inamdar, 2013). Son relevantes los resultados derivados de los trabajos de Regalado-Méndez donde se ha destacado en la estrategia enseñanza-aprendizaje seguida en el ABP la integración de conocimientos de varias materias. Así en la Universidad del Mar, México, se contrastó el éxito del ABP en estudiantes de grado en ingeniería ambiental que integraron conocimientos de informática, álgebra lineal y bioquímica (Regalado-Méndez et al., 2010b). Y en el ámbito de la ingeniería química también se ha aplicado el ABP integrando los conocimientos de varias asignaturas contrastándose un menor número de fracasos en el alumnado (Regalado-Méndez et al., 2010a). Se puede decir que actualmente la implantación del ABP en ingeniería se encuentra viviendo momentos similares a los ya acontecidos en el ámbito de la medicina en los años 60: numerosas pruebas y evaluaciones se han realizando mientras otras siguen su curso (Loncar-Vickovic et al., 2008; Mgangira, 2003; Vega et al., 2014).

Por otra parte, algunos trabajos han puesto el acento en aspectos concretos, como por ejemplo el efecto producido en los alumnos implicados (Yadav et al., 2011).También el ABP se ha extendido a otros campos de aplicación, más allá de la medicina y la ingeniería: por ejemplo las ciencias económicas (Santillán, 2006), la química (Lorenzo et al., 2011) e incluso la geografía (Latasa et al., 2012) han sido objeto de diversos trabajos de referencia. En cuanto a los resultados obtenidos han sidovariables (Ribeiro, 2005), y la puesta en práctica ha implicado en algunos casos variantes que han incluido apoyos al papel del profesor como facilitador empleando ordenadores (Mercier y Frederiksen, 2007). Como se puede comprobar por los antecedentes mencionados, y que suponen únicamente una breve selección de trabajos, el campo de investigación relacionado con el ABP, tanto directa como transversalmente, es amplio.

En cuanto a la posible ponderación del efecto que produce un ABP en los estudiantes, algunos trabajos han sido enfocados a aspectos distintos de la mera adquisición de conocimientos, como por ejemplo los valores y actitudes en el ámbito de la medicina (Carrión et al., 2015), o el desarrollo de competencias específicas en el ámbito de la ingeniería (Fernández y Duarte, 2013). En el presente trabajo el enfoque es en el rendimiento académico de los alumnos expresado por la superación en primer término, y la calificación 
máxima obtenida en segundo término, de la asignatura implicada en la evaluación. La herramienta de evaluación en este caso exige una adaptación conforme a los grupos en experimentación (Rodríguez, 2014). En este sentido podemos mencionar también la investigación realizada por Latasa et al. (2012), como afín en lo que corresponde a tamaños de grupos de evaluación del ABP y al análisis de las calificaciones finales obtenidas por los sujetos participantes.

Como antecedentes particulares y emparentados directamente con el trabajo efectuado, ya durante el curso 2013-14 se realizó una prueba piloto al respecto (Rodríguez, 2014). Comprendieron varias asignaturas del área de Ingeniería de la Construcción de la Universidad de Huelva, con docencia en varias titulaciones de ingeniería. Tras detectarse algunos fallos en la aplicación e implantación del ABP se decidió volver a realizar el experimento con la experiencia previa. El estudio piloto concluyó que se requería de un periodo previo con tratamiento expositivo tradicional (en adelante MET), pues aplicar directamente el ABP sin una fundamentación de conocimientos técnicos adecuados ralentizaba el proceso enseñanza-aprendizaje. También se determinaron aspectos de contexto necesarios, como aulas adecuadas al ABP y un tamaño de grupos de trabajo no mayor de 3 ó 4 alumnos.

En cuanto a la relación del ABP con el Aprendizaje Basado en Proyectos, refiriéndonos en nuestro caso a ingeniería y arquitectura (en adelante $\mathrm{ABPr}$ ), ambas son metodologías activas, aunque existen algunas diferencias. Para algunos autores (Mettas y Constantinou, 2008) consideran que el $\mathrm{ABPr}$ es una metodología activa donde el enfoque se realiza sobre un producto final, diseño o proyecto a desarrollar; en definitiva, una idea que se pretende hacer realidad. Según Rodríguez-Sandoval y Cortés-Rodríguez (2009) el ABPr se fundamenta en un proyecto el cual es desarrollado a lo largo de la impartición de la asignatura en cuestión, siendo ésta la esencia de su estrategia pedagógica. Se puede decir, por tanto, que el $\mathrm{ABPr}$ tiene siempre (y es un factor que lo condiciona) un eje central: el proyecto en sí. El ABPr, desde el punto de vista de los autores, es especialmente adecuado a la enseñanza-aprendizaje de materias en las cuales el diseño juegue un papel fundamental, como por ejemplo, en asignaturas de proyectos de carreras técnicas. En el caso de las construcciones agrarias, el ABP es, desde el punto de vista de los autores, más versátil que el ABPr pues el primero no está ceñido a un proyecto únicamente sino a problemas asociados a casos prácticos, incluyendo problemas de maquinaria, instalaciones, y ejecución de obras. En cualquier caso, aunque $A B P$ se refiera en su denominación a problemas y ABPr a proyectos, en el ámbito de la ingeniería no son metodologías mutuamente excluyentes, por lo que las apreciaciones sobre sus semejanzas y diferencias deben entenderse con flexibilidad.

Para finalizar esta introducción, mencionamos que el objetivo del estudio que se presenta ha sido determinar si existen diferencias significativas en los aprendizajes en construcciones agrarias entre alumnos que han participado en un programa de enseñanza basada en un ABP, y otros alumnos que han participado en un MET exclusivamente.

\section{MATERIAL Y MÉTODOS}

Comprende este apartado la enumeración de las líneas principales de actuación seguidas en el desarrollo de la investigación realizada junto a los factores que componen la estrategia de enseñanza-aprendizaje seguida. El problema de investigación se ha estructurado en 3 fases principales: 1) diseño de un ABP aplicado a la asignatura de construcciones agrarias; 2) aplicación y puesta en práctica del ABP y 3) evaluación del ABP con una técnica estadística no paramétrica. Estas fases principales requieren de ciertas operaciones. En la figura 1. Fases y operaciones de la investigación, se resumen en forma de esquema. A continuación se comenta la estrategia de enseñanza-aprendizaje, las fases y las operaciones:

\section{Estrategia de enseñanza-aprendizaje seguida}

En forma secuencial se consideró los siguientes factores clave: 1) Un aprendizaje en construcciones agrarias centrado en el alumno; 2) La generación del aprendizaje en grupos pequeños; 3) El aprendizaje radica en la generación de problemas; 4) El docente adquiere el papel de facilitador; 5) Los problemas generan habilidades; y 6) El aprendizaje autodirigido genera nuevo conocimiento. Los puntos que componen la estrategia adoptada han tenido en cuenta las apreciaciones de Barrows y Tamblyn (1980), y las precisiones fijadas para un auténtico ABP dadas por Woods (2012). La adaptación de la estrategia de enseñanza-aprendizaje al ámbito de la ingeniería de la construcción con empleo de ABP es propia de los autores y no debe entenderse tradicional.

1) Un aprendizaje en construcciones agrarias centrado en el alumno, donde estos deben tomar la responsabilidad de su propio aprendizaje, bajo la guía de un tutor. Se ha potenciado la personalización del aprendizaje del alumno, permitiéndole concentrarse en las áreas de conocimiento de interés para resolver el problema planteado. 
2) La generación del aprendizaje en grupos pequeños: los grupos de trabajo, que habitualmente se forman con 5 a 8 estudiantes en los ámbitos de las ciencias, los autores recomiendan en ingeniería 3 ó 4 alumnos por grupo por la experiencia adquirida previamente (Rodríguez, 2014; Rodríguez y Batanero, 2016). Al finalizar cada caso práctico (en total 11) los estudiantes cambian de grupo y trabajan con un nuevo grupo, permitiéndoles adquirir práctica con una variedad de diferentes personas.

3) El aprendizaje radica en la generación de problemas: en el ABP para construcciones agrarias se les ha planteado varios problemas sobre 11 casos prácticos, y se presenta a los estudiantes en un determinado formato un enunciado (papel o digital), al que acompaña una guía (previamente disponible en el repositorio digital) y que deben llevar a las aulas. La problemática propuesta representa el desafío que los estudiantes enfrentarán en la práctica y proporciona la relevancia y la motivación para el aprendizaje. Con el propósito de entender el problema, los estudiantes identifican lo que ellos tendrán que saber y comprender de las ciencias tecnológicas aplicadas. Como ejemplo, en la figura 2 se acompaña una parte de la documentación gráfica de un caso de estudio (el №7) que comprendió la construcción de una nave industrial para almacén de fruta en una zona franca de Cádiz (España). El caso de estudio en cuestión es real y la autoría corresponde al profesor responsable de la asignatura.

4) El docente adquiere el papel de facilitador. El profesor, que asume el rol de tutor, ha planteado preguntas a los estudiantes para encontrar por ellos mismos la mejor ruta de entendimiento y manejo del problema. Por ejemplo, en el caso práctico №7 (figura 1), entre otros problemas que se plantearon, se fijó encontrar una solución variante a la sección $A-A^{\prime}$ que permitiera una solera a la misma cota de rasante para los dos módulos de la nave de almacenamiento. El tutor va progresivamente "liberando" la solución que conlleva, en este caso, el diseño y cálculo de un muro de contención.

5) Los problemas generan habilidades. Para las disciplinas ingenieriles, es necesaria la presentación de un problema del mundo real o lo más cercano posible a una situación real, relacionada con aplicaciones del contexto profesional en el que el estudiante se desempeñará en el futuro. Por ejemplo, el hormigón armado se rige por una norma básica para cada país y unos componentes y características propias a cada tipo de hormigón; pero su aplicación real diferirá según los problemas propios de cada especialidad. Los procedimientos constructivos variarán, y las soluciones óptimas diferirán. Por tanto, los problemas han sido aplicados para la especialidad de ingeniería agrícola.

6) El aprendizaje autodirigido genera nuevo conocimiento. Durante este aprendizaje autodirigido, los estudiantes han trabajado juntos, discutiendo y comparando soluciones ingenieriles. Se espera que los estudiantes hayan aprendido a partir del conocimiento asociado a los casos prácticos, pero también se incrementará en el futuro en el mundo real con la acumulación de experiencia profesional. Este aspecto del $\mathrm{ABP}$ es para los futuros ingenieros quizás el más importante por el saber-hacer que ello procura.

\section{Diseño de un ABP para Construcciones Agrarias}

El análisis de trabajos previos comprende el estudio de anteriores investigaciones en el ámbito de la didáctica en general, y del ABP en particular. En cuanto al diseño del ABP para Construcciones Agrarias conlleva una detallada programación de la asignatura por semanas siendo recogida dicha programación (con las pruebas de control fijadas) en la correspondiente guía docente de la asignatura. Es imprescindible un periodo de 6 semanas de aplicación de un MET común a todos los alumnos, según se determinó en un estudio piloto anterior (Rodríguez, 2014). El citado estudio piloto se efectuó en el curso 2013-14 sobre 46 estudiantes de ingeniería de la construcción de las ramas de ingeniería agrícola e ingeniería minera. En este periodo es donde se exponen los fundamentos requeridos de la materia impartida. El apoyo de una plataforma virtual se hace necesario para no demorar el periodo común de MET de 6 semanas. Además, dicha plataforma tiene uso de repositorio digital, si bien es una herramienta más amplia que facilita una rápida intervención en las comunicaciones por parte del profesor-tutor y alumnado. A su vez, sirve de nexo de unión con otros ciberespacios de interés, siendo especialmente útil en la enseñanza de los procedimientos constructivos. El trabajo autónomo del estudiante independiente de las horas de clase se revela, por tanto, significativo. Las materias impartidas mediante un MET en el grupo de control se encuentran en forma implícita en los diferentes casos prácticos analizados en las correspondientes sesiones de ABP siendo comentadas en la solución final dada por el tutor una vez resuelto el caso en cuestión.

\section{Asignación de grupos experimental y de control}

El proceso de asignación de cada alumno a cada uno de los grupos ha seguido unas pautas que se exponen a continuación. Cada alumno ha elegido uno y sólo un grupo atendiendo a diferentes preferencias de horarios disponibles, sin tener en cuenta la metodología didáctica que iban a recibir pues no se ha conocido a priori. Las últimas plazas de cada grupo han exigido cierto proceso de negociación, atendiendo a interferencias de horarios con las prácticas de otras asignaturas y al número equilibrado de alumnos que se ha exigido entre los dos grupos formados. Una vez cerrado cada grupo (en total dos), se procedió a la 
asignación de cada grupo a grupo de control (GC) o experimental (GE) de forma aleatoria con una moneda al aire. El temario para ambos grupos fue el mismo, pero el grupo de control recibió únicamente MET mientras el grupo experimental recibió MET durante un periodo de tiempo de 6 semanas y ABP posteriormente durante 10 semanas. El periodo común para los dos grupos de 6 semanas de MET comprendió la base de conocimientos sobre materiales de construcción, repaso de los fundamentos de mecánica vectorial aplicada a la ingeniería, inicio de resistencia de los materiales y tipologías estructurales. Posteriormente se desglosaron las formas didácticas, siendo el ABP a partir de entonces aplicado al grupo experimental únicamente. El grupo de control continuó con el MET sin solución de continuidad temporal, incluyendo procedimientos constructivos, aplicaciones constructivas y ejercicios prácticos realizados por el docente sobre construcción y gestión de obras agrarias.

En cuanto al número de alumnos implicados en la investigación, han sido en total 52 . Es un número de alumnos adecuado a los medios docentes disponibles y a la atención en detalle que requiere un auténtico ABP. No todos los alumnos que han cursado la asignatura han participado de los grupos objeto de evaluación, existiendo un porcentaje en torno a un 10\% (habitualmente repetidores de la asignatura) que no asistieron regularmente a clase, quedando eliminados de las pruebas correspondientes. El tamaño de cada grupo se fijó en 26 alumnos. El total de matriculados en la asignatura ha ascendido a 60 alumnos para el curso 2015-16.

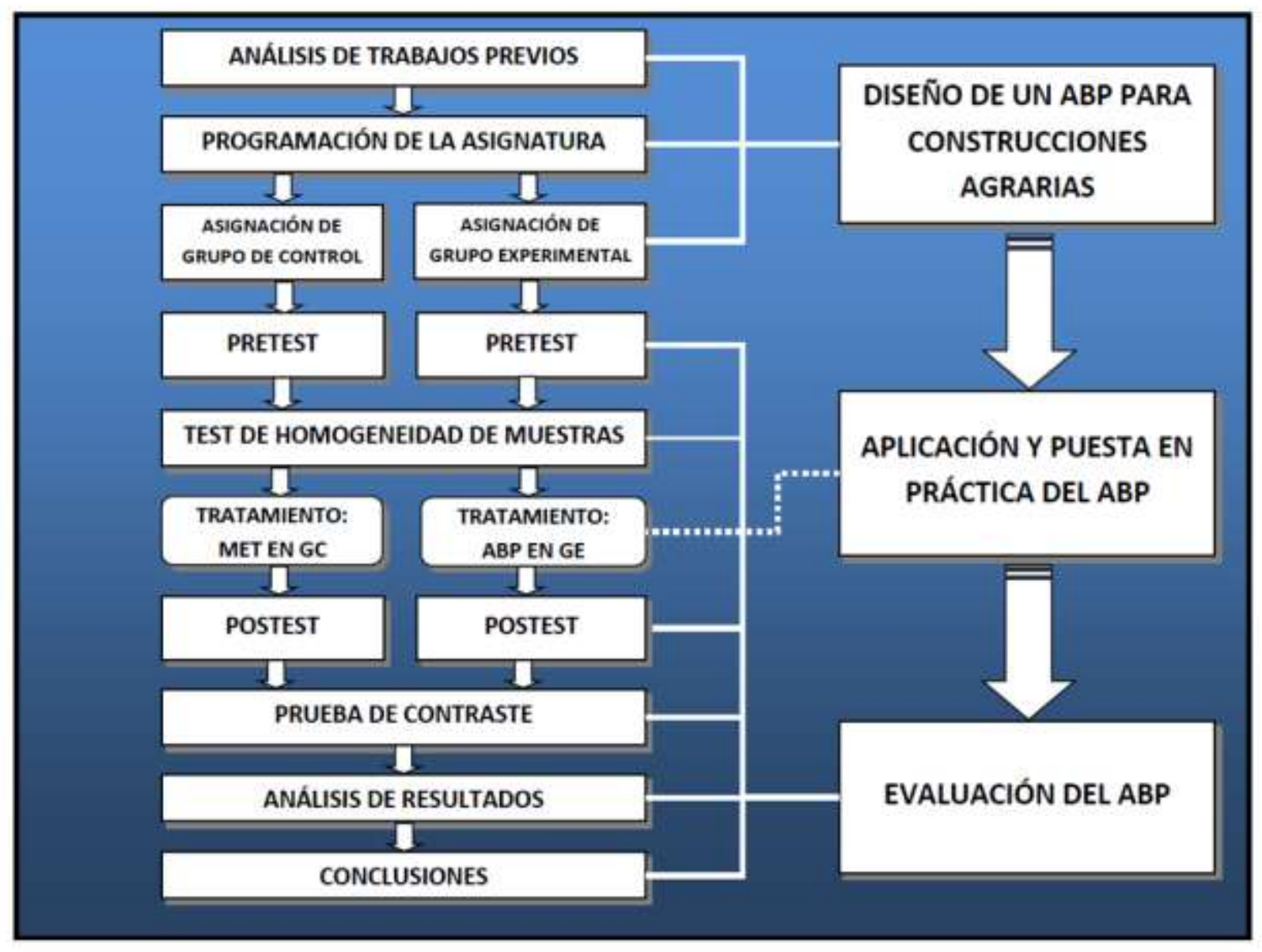

Fig.1. Fases y Operaciones de la Investigación.

Instrumento de medición y test de la mediana

El instrumento de medición ha consistido en pruebas objetivas para evaluación de conocimientos similares para los grupos experimentales y de control. Las pruebas objetivas se ajustan a la evaluación de conocimientos adquiribles y según las reglas establecidas en la guía docente de la asignatura en cuestión, publicada y revisada anualmente en la página web de la Universidad de Huelva. La objetividad responde a la herramienta empleada en la valoración final del examen del alumno, pues es la misma para todos. En el área de Ingeniería de la Construcción de la Universidad de Huelva y tras diez años aproximando una herramienta de valoración lo más objetiva posible, se llegó a la decisión de valorar no sólo los resultados numéricos, sino también los planteamientos, el orden y limpieza de los manuscritos, razonamiento lógico y conocimientos teóricos. 
De esta forma, las pruebas se estructuran en una parte teórica y una parte práctica, y su corrección responde a una fórmula (1) que se emplea en el área de Ingeniería de la Construcción para varias asignaturas, ponderando de forma diferente unos coeficientes que dependen de la materia concreta objeto de valoración. En la valoración final se incluye una ponderación que incluye tanto los resultados numéricos obtenidos en los problemas propuestos, como su planteamiento y otros aspectos formales. La parte teórica se evalúa con un examen tipo test. En la parte teórica los alumnos no pueden emplear apuntes ni libros para consulta. La parte práctica consiste en abordar un caso práctico ingenieril del ámbito de conocimientos de la asignatura en cuestión. En la parte práctica se han permitido apuntes, libros y material de consulta, si bien no se ha permitido contacto verbal entre los estudiantes en ninguna de las partes en que se subdivide la prueba en cuestión. La corrección de las pruebas objetivas y la correspondiente asignación de una nota numérica emplea la siguiente expresión:

$R=K_{1} \cdot\left(\frac{10 \cdot A-3,3 \cdot F}{n}\right)+K_{2} \cdot(0,70 \cdot V+0,20 \cdot P+0,10 \cdot S)$

Siendo $R$ el resultado final de la prueba, de 0 a 10 siendo 5 el aprobado; $K_{1}$ el coeficiente de ponderación de la parte teórica (para Construcciones Agrarias 0,30 ); $K_{2}$ el coeficiente de ponderación de la parte práctica (para Construcciones Agrarias 0,70); $n$ el número de preguntas del test teórico (10 ítems para Construcciones Agrarias con cuatro respuestas posibles y una única posible correcta); $A$ es el número de respuestas acertadas en el test de la parte práctica; $F$ es el número de respuestas erróneas en el test de la parte práctica; $V$ es la valoración del resultado final obtenido en la parte práctica (de 0 a 10 ); $P$ es la valoración de los planteamientos de los problemas de la parte práctica (de 0 a 10) y $S$ es la valoración de los aspectos formales de la parte práctica como son el orden y cuidado en la exposición, limpieza y razonamiento lógico (de 0 a 10).

La técnica no paramétrica seleccionada para el contraste de hipótesis ha sido la prueba de la mediana, variante de la prueba $\chi^{2}$, adaptada en nuestro caso para medida ordinal y adecuada al tamaño de la muestra y al tipo de datos con los que se ha trabajado. El tamaño de los grupos impone técnicas no paramétricas para su análisis estadístico. En cuanto a la selección concreta de la técnica en cuestión, el empleo de la técnica de la mediana está aconsejado en contrastes de hipótesis para estudios con diseño similar según las recomendaciones expuestas por autores solventes en la materia (García Llamas et al., 2006).

\section{Variables definidas y test de homogeneidad de muestras}

Se han realizado pretest en ambos grupos al efecto de comprobar si son comparables los grupos en cuestión. Resultó que no existen diferencias significativas aplicando el test de la mediana al ser $\chi^{2}$ teórico = $3,841>0,696 \chi^{2}$ empírico, para un nivel de confianza del $95 \%$, con lo cual han podido ser comparables los grupos definidos. Se estableció, tanto para el pretest como para el postest una cláusula ceteris paribus para toda la investigación empírica. En este sentido, la variable dependiente es el rendimiento académico y la independiente el tratamiento mediante ABP o MET. Formalmente: haber sido o no sujeto sometido a tratamiento durante 10 semanas (ABP en nuestro caso) es la variable independiente. En la evaluación, la hipótesis a falsar o verificar es "el tratamiento dado mediante ABP no afecta al rendimiento académico".

Como medidas al respecto, se han adoptado las siguientes: 1) el pretest y postest lo han efectuado los grupos experimental y de control a la vez y en el mismo lugar; 2) ambos grupos, experimental y de control, han efectuado pruebas similares; 3 ) la corrección de exámenes ha sido triangulada por otro profesor con docencia en el área distinto del que imparte la docencia en el grupo en cuestión, y 4) Los resultados finales han sido validados mediante un juicio de expertos (tres profesores de más antigüedad con docencia en ingeniería, distintos del que imparte la docencia en el grupo y distinto del profesor del área que triangula la corrección de exámenes). Como variables extrañas, que no se han podido controlar y que han podido afectar al experimento están: 1) lugar de impartición de las clases; 2) horario de las clases variable por grupo y 3 ) interferencia de festividades para cada grupo diferente. Razones operativas han hecho imposible controlar las variables extrañas mencionadas. No obstante, se ha invertido un esfuerzo en que su interferencia haya sido la menor posible.

\section{Evaluación del ABP mediante un método cuasi-experimental}

Con respecto a la evaluación del $\mathrm{ABP}$, se ha tenido en cuenta tanto aspectos de eficacia como de eficiencia, siendo la primera expresada por el mayor número de aprobados, y la eficiencia por la mayor calificación obtenida para un mismo nivel de recursos empleados. El modelo empleado para la evaluación del ABP ha consistido en un modelo con un diseño cuasiexperimental con grupos de control y experimental no equivalente. 


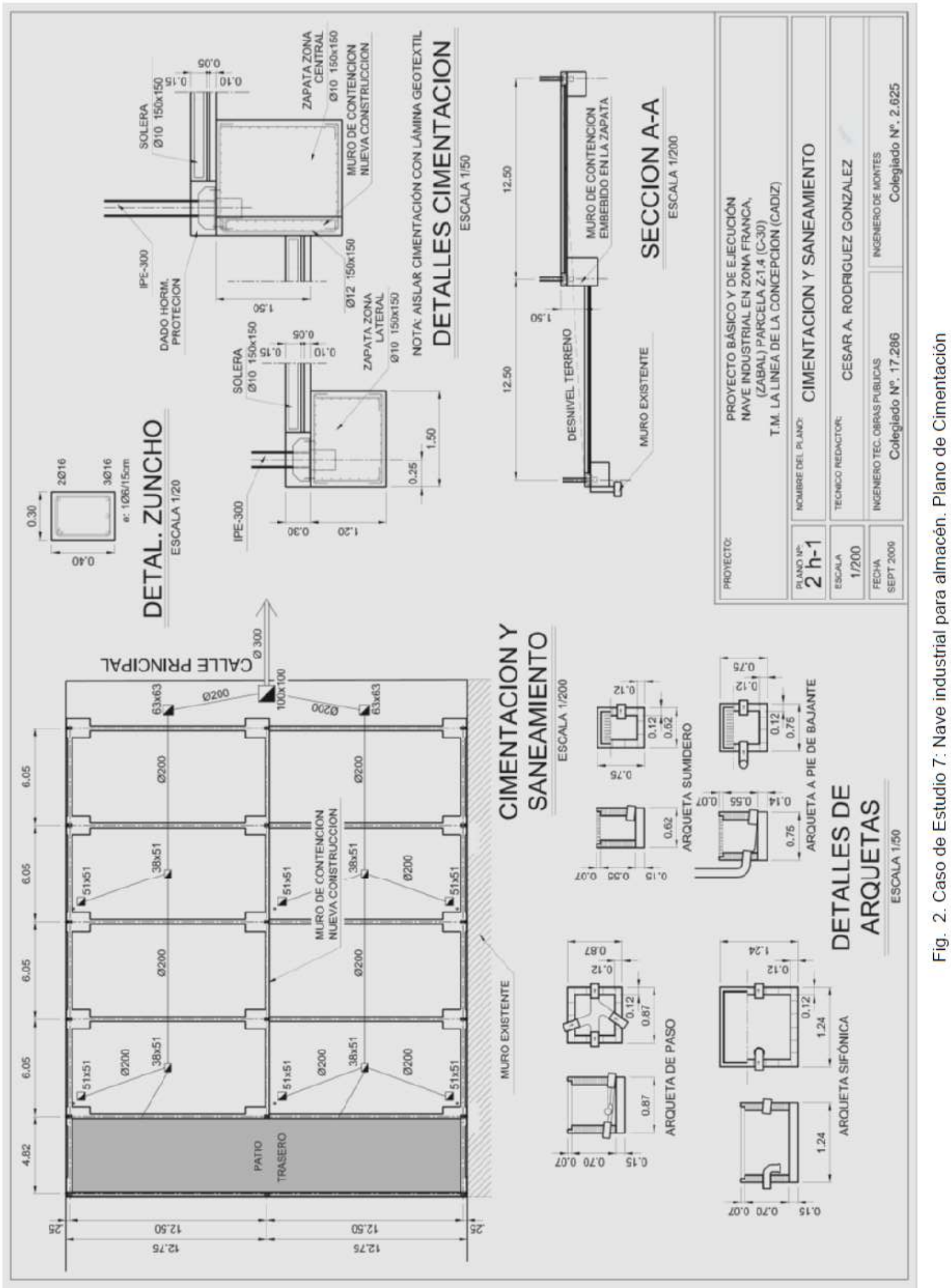

En lo que concierne al carácter del modelo, desde el punto de vista del método científico, está enmarcado dentro de los modelos nomológico-inductivos, cuyo fundamento consiste en falsar o aceptar una hipótesis de partida aplicada a una muestra y establecer las correspondientes inducciones. Las conclusiones que se 
pueden deducir sobre el resultado obtenido sobre la citada muestra se pueden inducir para una población mayor con ciertas limitaciones.

El número de alumnos limita la exactitud alcanzable en este sentido, dado que no se puede garantizar una normalidad perfecta en las muestras empleadas. Sin embargo, precisamente es el número de alumnos por grupo no mayor a 30 el que permite aplicar un ABP auténtico y con las exigencias de tutorización que requiere. En lo que a fundamentos básicos del método cuasiexperimental aplicado al ámbito de la educación se han tenido en cuenta los planteamientos y ejemplos de García Llamas, Pérez Juste y Río Sadornil (2006).

\section{RESULTADOS Y DISCUSIÓN}

Para comprobar si existe una mejora significativa del rendimiento académico una vez recibido un tratamiento mediante $\mathrm{ABP}$, se empleó la técnica no paramétrica del test de la mediana comentado, debido al reducido tamaño de las muestras (inferiores cada grupo a 30 alumnos). Se hicieron las pruebas de control (pretest y postest) y se obtuvieron las siguientes calificaciones mostradas en la tabla 1.

Con respecto a la evaluación, los puntos que definen la prueba efectuada son: 1) diseño cuasiexperimental, de dos grupos independientes, con una variable independiente y muestras pequeñas $(n<30)$; 2$)$ nivel de medida ordinal; 3) hipótesis bilateral: a) $\mathrm{H}_{0}$ : no existen diferencias entre ambos grupos y b) $\mathrm{H}_{1}$ : existen diferencias significativas; 4) contraste no paramétrico. Prueba de la mediana, puesto que la medida empleada hace referencia a dos únicos rangos o categorías: por encima o por debajo de la mediana o lugar central de todas las puntuaciones y 5) pasos: a) obtención de la mediana de todas las puntuaciones. $\mathrm{Md}=$ 5,8 b) obtención de un $\chi^{2}$ teórico para un nivel de confianza del $95 \%$ y 1 grado de libertad $\left(\chi^{2}\right.$ teórico $\left.=3,841\right)$ y c) obtención de un $\chi^{2}$ empírico mediante la siguiente expresión (2) que relaciona las frecuencias observadas $f_{o}$ y las frecuencias esperadas $f_{e}$ :

Tabla 1. Resultados de la prueba de control de conocimientos por grupos. Orden acorde al test de la mediana.

\begin{tabular}{cccccccc}
\hline \multicolumn{3}{c}{ Pretest } \\
\hline \multicolumn{2}{c}{ Grupo de Control } & \multicolumn{3}{c}{ Grupo Experimental } & Grupo de Control & Grupo Experimental \\
\hline 1,6 & 4,0 & 1,3 & 4,5 & 2,0 & 5,0 & 1,3 & 7,2 \\
2,0 & 4,0 & 2,0 & 4,7 & 2,2 & 5,0 & 2,5 & 7,4 \\
2,2 & 4,0 & 2,0 & 4,8 & 2,3 & 5,3 & 3,0 & 7,5 \\
2,5 & 4,2 & 2,4 & 5,0 & 2,5 & 5,5 & 3,3 & 7,7 \\
2,8 & 4,4 & 2,6 & 5,0 & 3,0 & 5,7 & 3,7 & 8,0 \\
2,8 & 4,5 & 2,6 & 5,0 & 3,3 & 5,8 & 4,0 & 8,0 \\
3,0 & 4,5 & 2,8 & 5,2 & 3,4 & 6,0 & 4,5 & 8,0 \\
3,2 & 4,5 & 3,5 & 5,3 & 3,7 & 6,0 & 5,0 & 8,2 \\
3,3 & 4,7 & 3,5 & 5,5 & 4,0 & 6,1 & 5,0 & 8,4 \\
3,4 & 5,0 & 3,7 & 5,6 & 4,2 & 6,2 & 6,0 & 8,5 \\
3,5 & 5,5 & 3,8 & 5,6 & 4,5 & 6,5 & 6,6 & 8,5 \\
3,7 & 5,7 & 4,0 & 6,0 & 5,0 & 7,0 & 6,8 & 8,7 \\
3,8 & 6,5 & 4,2 & 6,2 & 5,0 & 7,2 & 7,0 & 9,0 \\
\hline
\end{tabular}

$\chi^{2}=\sum_{g=1}^{G} \sum_{c=1}^{C} \frac{\left(\left|f_{o}-f_{e}\right|-0,5\right)^{2}}{f_{e}}$

Se comprobó que al ser $\chi^{2}$ teórico $=3,841<4,930 \chi^{2}$ empírico, hay diferencias significativas entre ambos grupos, a nivel de postest y para un nivel de confianza del 95\%. Por tanto, se rechaza entonces la hipótesis nula $\left(\mathrm{H}_{0}\right)$ y se acepta la hipótesis alternativa $\left(\mathrm{H}_{1}\right)$ : existen diferencias significativas en el rendimiento académico al recibir ABP frente a recibir otro método tradicional.

De los datos anteriores se deriva la nota media en ambos grupos, habiendo sido de 4,7 en el grupo de control y 6,3 en el grupo experimental. Podemos deducir que existe una armonización con los resultados de trabajos previos sobre ABP, reduciéndose el número de suspensos (Regalado-Méndez et al., 2010a) e incrementándose los resultados de aprendizaje positivos con respeto al MET (Latasa et al., 2012). Las 
desviaciones típicas han sido a su vez de 1,53 y 2,22 respectivamente. Y el porcentaje de aprobados ha sido del $58 \%$ y $73 \%$ respectivamente para los grupos de control y experimental. Se deduce de ello que los grupos que reciben ABP tienen mayor nota media, pero también mayor variabilidad en sus resultados. En el nivel de conocimientos actual y con los datos disponibles, se puede deducir una mejora en el rendimiento académico del grupo que recibió $\mathrm{ABP}$ frente al grupo que recibió únicamente MET.

\section{CONCLUSIONES}

Se concluye que: 1) existen diferencias significativas en los aprendizajes en construcciones agrarias entre alumnos que han participado en un programa de enseñanza basada en un ABP, y otros alumnos que han participado en un programa de aprendizaje siguiendo un MET. Se deduce, por tanto, que el ABP es efectivo con un periodo de 10 semanas de aplicación; 2) el ABP se configura como un método didáctico eficaz, y a su vez, más eficiente que el MET. La mayor nota media y el mayor número de aprobados lo evidencian; y 3 ) el ABP se configura como un método didáctico eficaz, y a su vez, más eficiente que el método que combina el expositivo y de realización de problemas por docente. La mayor nota media y el mayor número de aprobados lo evidencian.

\section{REFERENCIAS}

Barrows, H. S., How to design a problem-based curriculum for the preclinical years, Vol.8, Springer Pub. Co. New York, USA (1985)

Barrows, H. S. y R. M. Tamblyn, Problem-based learning: an approach to medical education, Springer Publishing Company, New York, USA (1980)

Branda, L. A., El aprendizaje basado en problemas. De heregía artificial a res popularis, http://scielo.isciii.es/scielo.php?script=sci_arttext\&pid=S1575-18132009001100004, ISSN: 1575-1813, Educación Médica, 12 (1), 11-23 (2009)

Carrion, C.; M. Soler y M. Aymerich, Análisis de la Validez de Contenido de un Cuestionario de Evaluación del Aprendizaje Basado en Problemas. Un Enfoque Cualitativo, doi: 10.4067/S0718-50062015000100003, Formación Universitaria, 8(1), 13-22 (2015)

Felder, R. M. y R. Brent, Active learning: An introduction, ASQ Higher Education Brief, 2(4), 1-5 (2009)

Fernández, F.H. y J.E. Duarte, El aprendizaje basado en problemas como estrategia para el desarrollo de competencias específicas en estudiantes de ingeniería, doi: 10.4067/S0718-50062013000500005, Formación Universitaria, 6 (5), 29-38 (2013)

García Llamas, J.L.; R. Pérez Juste y D. Río Sadornil, Problemas y diseños de investigación resueltos (3a edición revisada y ampliada), Dykinson, Madrid, España (2006)

Latasa, I.; P. Lozano y N. Ocerinjauregi, Aprendizaje basado en problemas en currículos tradicionales: Beneficios e inconvenientes, doi: 10.4067/S0718-50062012000500003, Formación Universitaria, 5(5), 15-26 (2012)

Loncar-Vickovic, S.; Z. Dolacek-Alduk y D. Stober, Use of problem-based learning in higher education: student workshops at the faculty of civil engineering in osijek, http://hrcak.srce.hr/index.php?show=clanak\&id_clanak_jezik=49804, ISSN: 1330-3651, Tehnicki VjesnikTechnical Gazette, 15(4), 35-40 (2008)

Lorenzo, R.A., P. Fernández y A.M. Carro, Experiencia en la Aplicación del Aprendizaje Basado en Problemas en la Asignatura Proyecto de Licenciatura en Química, doi: 10.4067/S071850062011000200005, Formación Universitaria, 4(2), 37-44 (2011)

Mercier, J. y C.H. Frederiksen, Individual differences in graduate students' help-seeking process in using a computer coach in problem-based learning, doi: 10.1016/j.learninstruc.2007.01.013, Learning and Instruction, 17(2), 184-203 (2007)

Mettas, A.C. y C.C. Constantinou, The technology fair: a project-based learning approach for enhancing problem solving skills and interest in design and technology education, doi: 10.1007/s10798-006-9011-3, International Journal of Technology and Design Education, Dordrecht, The Netherlands, 18 (1), 79-100 (2008) 
Mgangira, M. B., Integrating the development of employability skills into a civil engineering core subject through a problem-based learning approach, International Journal of Engineering Education, 19(5), 759-761 (2003)

Regalado-Méndez, A., M.R.P. Cid-Rodríguez y J.G. Báez-González, Problem Based Learning (PBL): Analysis of Continuous Stirred Tank Chemical Reactors with a Process Control Approach, doi: 10.5121/ijsea.2010.1404, International Journal of Software Engineering \& Applications (IJSEA): 1(4), 54-73 (2010a)

Regalado-Méndez, A. y otros tres autores, Problem Based Learning: Obtaining Enzyme Kinetics Parameters Integrating Linear Algebra, Computer Programming and Biochemistry Curriculum, doi: 10.1007/978-90-4819151-2_3, in Technological Developments in Networking, Education and Automation, pp 13-18, Springer Netherlands (2010b)

Ribeiro, L. R. D., y M.D.N., Mizukami, Student assessment of a problem-based learning experiment in civil engineering education, doi: 10.1061/(ASCE)1052-3928(2005)131:1(13), Journal of Professional Issues in Engineering Education and Practice, 131(1), 13-18 (2005)

Rodríguez, C.A., Diseño, aplicación y validación de un ABP en estudiantes universitarios de ingeniería de la construcción, Tesina de maestría, Facultad de Ciencias de la educación, Universidad de Sevilla (2014)

Rodríguez, C.A. y J.M. Fernández-Batanero, A review of Problem-Based Learning applied to Engineering, http://hdl.handle.net/11441/41227, ISSN: 2340-2504, EduRe Journal: International Journal on Advances in Education Research, 3 (1), 14-31 (2016)

Rodríguez-Sandoval, E. y M. Cortés-Rodriguez, Evaluación de la estrategia pedagógica "aprendizaje basado en proyectos": percepción de los estudiantes, http://www.scielo.br/pdf/aval/v15n1/v15n1a08, ISSN: 1982-5765, Avaliação: Revista da Avaliação da Educação Superior, 1(15), 31-37 (2010)

Santillán, F., El Aprendizaje Basado en Problemas como propuesta educativa para las disciplinas económicas y sociales apoyadas en el B-Learning, http://rieoei.org/1460.htm, ISSN: 1681-5653, Revista Iberoamericana de Educación, 40/2, 1-5 (2006)

Shinde, V. V. y S.S. Inamdar, Problem Based Learning (PBL) for Engineering Education in India: Need and Recommendations, doi: 10.1007/s11277-013-1069-0, Wireless Personal Communications: An International Journal, 69(3), 1097-1105 (2013)

Vega, F., E. Portillo, M. Cano y B. Navarrete, Experiencias de aprendizaje en ingeniería química: diseño, montaje y puesta en marcha de una unidad de destilación a escala laboratorio mediante el aprendizaje basado en problemas, doi: 10.4067/S0718-50062014000100003, Formación Universitaria, 7(1), 13-22 (2014)

Woods, D. R., PBL: An Evaluation of the Effectiveness of Authentic Problem-Based Learning (aPBL), http://www.che.ufl.edu/cee/Journals/Spring\%202012\%20v46.2/Woods462.html, ISSN: 0009-2479, Chemical Engineering Education: 46(2), 135-144 (2012)

Yadav, A. y otros tres autores, Problem-based Learning: Influence on Students' Learning in an Electrical Engineering Course, doi: doi:10.1002/j.2168-9830.2011.tb00013.x, Journal of Engineering Education: 100(2), 253-280 (2011) 